\title{
LIMIT THEOREMS FOR RANDOM TRIANGULAR URN SCHEMES
}

\author{
RAFIK AGUECH, ${ }^{*}$ Faculté des Sciences de Monastir, Tunisia
}

\begin{abstract}
In this paper we study a generalized Pólya urn with balls of two colors and a random triangular replacement matrix. We extend some results of Janson (2004), (2005) to the case where the largest eigenvalue of the mean of the replacement matrix is not in the dominant class. Using some useful martingales and the embedding method introduced in Athreya and Karlin (1968), we describe the asymptotic composition of the urn after the $n$th draw, for large $n$.
\end{abstract}

Keywords: Multitype branching process; generalized Pólya urn; urn model; Yule process

2000 Mathematics Subject Classification: Primary 60J80; 60J85; 60G40; 62G20

Secondary 60K05; 60G46; 60F05

\section{Introduction}

We consider a generalized Pólya urn with balls of two colors, say white (W) and black (B). The urn is initially nonempty. At each time $n$, a ball is drawn from the urn uniformly at random and its color is observed (i.e. a ball is drawn, looked at, and then placed back into the urn). If a white ball is drawn then it is replaced in the urn with $X_{n}$ white balls; if a black ball is drawn then it is replaced in the urn with $Y_{n}$ white balls and $Z_{n}$ black balls. The random variables $X_{n}$, $Y_{n}$, and $Z_{n}$ are independent copies of some nonnegative, integer-valued random variables $X$, $Y$, and $Z$, respectively.

The evolution rule at time $n$ is then summarized by the $2 \times 2$ random matrix

$$
\mathrm{W}\left(\begin{array}{cc}
\mathrm{W} & \mathrm{B} \\
X_{n} & 0 \\
Y_{n} & Z_{n}
\end{array}\right) \text {, }
$$

where the rows indicate the number of balls added to the urn and the columns indicate the number of balls drawn. Thus, the composition of the urn after $n$ draws is represented by the vector $\left(W_{n}, B_{n}\right)$, where $W_{n}$ and $B_{n}$ are the numbers of white and black balls, respectively, in the urn. The urn starts with a given vector $\left(W_{0}, B_{0}\right)$, which we assume is nonrandom.

The assumption that $X, Y$, and $Z$ are nonnegative, integer-valued random variables guarantees the nonextinction of the urn. Furthermore, in order to avoid any explosion of the urn, we suppose that $X, Y$, and $Z$ have finite variances. We define

$$
\begin{array}{ccc}
\mu_{X}=\mathrm{E}(X), & \mu_{Y}=\mathrm{E}(Y), & \mu_{Z}=\mathrm{E}(Z), \\
\sigma_{X}^{2}=\operatorname{var}(X), & \sigma_{Y}^{2}=\operatorname{var}(Y), \quad \sigma_{Z}^{2}=\operatorname{var}(Z),
\end{array}
$$

Received 27 June 2007; revision received 6 July 2009.

* Postal address: Département de Mathématiques, Faculté des Sciences de Monastir, 5019 Monastir et EPAM Sousse, Tunisia. Email address: rafik.aguech@ipeit.rnu.tn 
and, for every integer $n$, the ball addition matrix or the replacement matrix is

$$
R(n)=\left(\begin{array}{cc}
X_{n} & 0 \\
Y_{n} & Z_{n}
\end{array}\right) .
$$

Eggenberger and Pólya [6] were among the first to study urn models, dealing with the fixed schemata

$$
\left(\begin{array}{ll}
s & 0 \\
0 & s
\end{array}\right) .
$$

This urn is known as the Pólya-Eggenberger urn. In 1931 this model was discussed by Pólya [15]. Bernstein [5] and Savkevitch [16] then generalized this model to the case where $s$ balls of the same color and $a$ balls of the antithetical color are added:

$$
\left(\begin{array}{ll}
s & a \\
a & s
\end{array}\right)
$$

These two models have been studied and generalized in later papers (see [1], [4], [7], [8], and [9]).

Several Pólya urn models with various settings for the ball addition matrix have been studied by many authors. In particular, in the case when the mean of the replacement matrix is irreducible, Janson [11] carried out a study in which he characterized the number of balls of each color. In the case when the replacement matrix is triangular, not irreducible, and nonrandom, Janson [12] characterized the limit law and the almost-sure limits of the number of balls of each color. The aim of this paper is to extend these results to the case when the replacement matrix is assumed to be random.

To attempt our goal, we will use the embedding method of Athreya and Karlin [2], [3]. We study the urn process by embedding it into a multitype continuous-time Markov branching process $\mathcal{X}(t)=(W(t), B(t))$ with initial condition $\mathcal{X}(0)=(W(0), B(0))=\left(W_{0}, B_{0}\right)$. In the continuous process we assume that every ball of type $i$ (where white balls are of type 1 and black balls are of type 2) has an exponentially distributed lifetime with mean 1, i.e. it dies with intensity 1 , and when it dies, it is replaced by a set of balls with distribution $\left(\delta_{i, j}\right)_{j=1,2}$, where, if we note by $\mathcal{L}$ the distribution,

$$
\delta_{1,1}=\mathcal{L}(X+1), \quad \delta_{1,2}=\mathcal{L}(0), \quad \delta_{2,1}=\mathcal{L}(Y), \quad \delta_{2,2}=\mathcal{L}(Z+1) .
$$

We shall assume that all lifetimes and offspring compositions are independent.

Let $0=\tau_{0}<\tau_{1}<\tau_{2}<\cdots<\tau_{n}<\cdots$ be the splitting times of the process $\mathcal{X}$. Athreya and Karlin [2] proved that the process $\left(\mathcal{X}\left(\tau_{n}\right)\right)_{n \geq 0}$ has the same law as $\left(\left(W_{n}, B_{n}\right)\right)_{n \geq 0}$; hence, since $\tau_{n} \stackrel{\text { a.s. }}{\rightarrow} \infty$ as $n \rightarrow \infty$, limit theorems for $\left(W_{n}, B_{n}\right)$ can be derived from limit theorems for $\mathcal{X}(t)$. By $\stackrel{\text { a.s. }}{\rightarrow}$ we denote almost-sure convergence.

In order to study the process $\mathcal{X}(t)=(W(t), B(t))$, we define a suitable martingale $\mathcal{Y}(t)$. The martingale that we use is a standard one in branching process theory. Let

$$
A:=\mathrm{E}\left(R^{\top}(n)\right)=\left(\begin{array}{cc}
\mu_{X} & \mu_{Y} \\
0 & \mu_{Z}
\end{array}\right),
$$

and define $y(t)=\mathrm{e}^{-t A} \mathcal{X}(t)^{\top}$, where $M^{\top}$ denotes the transpose of the matrix $M$. Let $\mathcal{F}_{t}$ be the $\sigma$-algebra generated by the family $\{(W(s), B(s)), s \leq t\}$.

Using the Markov property, in the following theorem we extend a fundamental, well-known result described in [11, Lemma 9.8], and [3, Theorem V.7.2]. The proof of this result will be relegated to Section 3 . 
Theorem 1. If $\mu_{X} \geq \mu_{Z}$, the martingale $\left\{\mathcal{y}(t), \mathcal{F}_{t}, t \geq 0\right\}$ is an $L^{2}$-bounded martingale, and, hence, converges almost surely and in $L^{2}$. Moreover, $\overline{\mathcal{Y}}(t)=\mathrm{E}\left(\widetilde{\mathcal{H}} / \mathcal{F}_{t}\right)$, where

$$
\widetilde{\mathcal{y}}:=\left(\begin{array}{c}
W \\
B
\end{array}\right)
$$

is the almost sure and $L^{2}$-limit of $\mathcal{y}(t)$. Here $W$ and $B$ are two random variables.

Remarks. 1. We still get an almost sure and $L^{1}$-convergence of the martingale $y(t)$ if we replace the assumption that $X, Y$, and $Z$ have finite variances by

$$
\mathrm{E}(X \ln X)+\mathrm{E}(Y \ln Y)+\mathrm{E}(Z \ln Z)<\infty .
$$

However, for our main results, we need the $L^{2}$-assumption.

2. Using Theorem 1, we can prove the following statements.

- If $\mu_{X}>\mu_{Z}$, we have

$$
\begin{aligned}
\exp \left(-t \mu_{X}\right) W(t)+\frac{\mu_{Y}}{\mu_{X}-\mu_{Z}}\left(\exp \left(-t \mu_{X}\right)-\exp \left(-t \mu_{Z}\right)\right) B(t) \\
\quad \rightarrow W \quad \text { almost surely and in } L^{2}, \\
\quad \exp \left(-t \mu_{Z}\right) B(t) \rightarrow B \text { almost surely and in } L^{2} .
\end{aligned}
$$

As proved in Theorem 3.1 of [11], we have the functional limit theorem. In $D[0, \infty)$, the space of all right-continuous functions $[0, \infty) \rightarrow \mathbb{C}$ with left-hand limits,

$$
\begin{gathered}
\exp \left(-t x \mu_{X}\right) W(t x)+\frac{\mu_{Y}}{\mu_{X}-\mu_{Z}}\left(\exp \left(-t x \mu_{X}\right)-\exp \left(-t x \mu_{Z}\right)\right) B(t x) \stackrel{\mathcal{L}}{\longrightarrow} W, \\
\quad \exp \left(-t x \mu_{Z}\right) B(x t) \stackrel{\mathcal{L}}{\longrightarrow} B .
\end{gathered}
$$

- If $\mu_{X}=\mu_{Z}$, we have

$$
\begin{gathered}
\exp \left(-t \mu_{X}\right) W(t)-\mu_{Y} t \exp \left(-t \mu_{X}\right) B(t) \rightarrow W \quad \text { almost surely and in } L^{2}, \\
\exp \left(-t \mu_{X}\right) B(t) \rightarrow B \quad \text { almost surely and in } L^{2},
\end{gathered}
$$

and, in $D[0, \infty)$,

$$
\begin{gathered}
\exp \left(-x t \mu_{X}\right) W(x t)-\mu_{Y} x t \exp \left(-x t \mu_{X}\right) B(x t) \stackrel{\mathcal{L}}{\longrightarrow} W, \\
\exp \left(-x t \mu_{X}\right) B(x t) \stackrel{\mathcal{L}}{\longrightarrow} B .
\end{gathered}
$$

By ' $\stackrel{\mathcal{L}}{\longrightarrow}$, we denote convergence in law.

3. By Theorem $1, B$ is a nonnegative random variable with $\mathrm{E}(B)=B_{0}$. Using Theorems III.4.1 and III.7.2 of [3], we deduce that

$$
\mathrm{P}(B=0)=0 .
$$

4. Let $g_{Z}(s)=s \mathrm{E}\left(s^{Z}\right)$ be the probability generating function of $Z+1$, and let $\varphi_{B}(u)=$ $\mathrm{E}\left(\mathrm{e}^{-u B}\right)$. Then (see [10] and [13])

$$
\varphi_{B}^{-1}(v)=(1-v) \exp \left(\int_{v}^{1}\left[\frac{1}{s-1}-\frac{\mu_{Z}}{g_{Z}(s)-s}\right] \mathrm{d} s\right), \quad 0<v \leq 1 .
$$

5. The author has been unable to characterize the distribution of $W$ in all cases.

Theorem 1 is the basis of all our results for the branching process and generalized Pólya urns. 
The paper is organized as follows. In Section 2 we give our main results after having specified the notation. Section 3 deals with the proof of Theorem 1. Finally, the proofs of the main results are given in Section 4.

\section{Main results}

In the following, $\mathcal{P}(\lambda)$ denotes a Poisson random variable with parameter $\lambda$ and $\mathcal{N}\left(\mu, \sigma^{2}\right)$ denotes the normal law with mean $\mu$ and variance $\sigma^{2}$. Let $B_{n}$ and $W_{n}$ denote the numbers of black and white balls, respectively, in the urn after $n$ draws, and let $B$ and $W$ denote the limiting random variables defined in Theorem 1 . For $n \geq 1$, we denote by $S_{n}$ the total number of balls in the urn after the $n$th draw. We have $S_{n}=W_{n}+B_{n}$ and $S_{0}=W_{0}+B_{0}$. Let $N_{n}^{\mathrm{W}}$ and $N_{n}^{\mathrm{B}}$ denote the numbers of white and, respectively, black balls drawn in the first $n$ draws. We have $N_{n}^{\mathrm{B}}+N_{n}^{\mathrm{W}}=n$. Let $\tau_{1}^{\mathrm{W}}<\cdots<\tau_{N_{n}^{\mathrm{W}}}^{\mathrm{W}}$ and $\tau_{1}^{\mathrm{B}}<\cdots<\tau_{N_{n}^{\mathrm{B}}}^{\mathrm{B}}$ be the splitting times, before $\tau_{n}$, of the $N_{n}^{\mathrm{W}}$ white balls and the $N_{n}^{\mathrm{B}}$ black balls, respectively. The basic hypotheses of this section are as follows (collectively referred to as $(\mathrm{H})$ ).

(H1) $X, Y$, and $Z$ are nonnegative, independent, integer-valued random variables satisfying
(i) $\sigma_{X}+\sigma_{Y}+\sigma_{Z}<\infty$,
(ii) $\mu_{X} \geq \mu_{Z}$,
(iii) $\mu_{X}\left(\mu_{Y}+\mu_{Z}\right)>0$.

(H2) The initial composition of the urn is $\left(W_{0}, B_{0}\right)$, with $B_{0}>0$.

The main results of this paper are Theorems 2-5 and Proposition 1, below.

Theorem 2. Consider a generalized Pólya urn with two colors and a triangular random replacement matrix

$$
\left(\begin{array}{ll}
X & 0 \\
Y & Z
\end{array}\right)
$$

Let

$$
\rho=\frac{\mu_{Z}}{\mu_{X}} \quad \text { and } \quad K=\left(\mu_{X}\right)^{\rho} B\left(W+\frac{\mu_{Y}}{\mu_{X}-\mu_{Z}} B\right)^{-\rho} .
$$

Under hypotheses $(H)$ and the fact that $\mu_{Y}>0$, we obtain the following results.

1. For $\mu_{X}>\mu_{Z}>0$,

(i) we have, almost surely,

$$
\begin{gathered}
W_{n}=\mu_{X} n+o(n), \quad B_{n}=K n^{\rho}+o\left(n^{\rho}\right), \\
N_{n}^{\mathrm{W}}=n-\frac{K}{\mu_{Z}} n^{\rho}+o\left(n^{\rho}\right), \quad N_{n}^{\mathrm{B}}=\frac{K}{\mu_{Z}} n^{\rho}+o\left(n^{\rho}\right),
\end{gathered}
$$

(ii) if $\rho \leq \frac{1}{2}$ then

$$
\frac{W_{n}-n \mu_{X}}{\sqrt{n}}-\frac{K}{\mu_{Z}}\left(\mu_{Y}-\mu_{X}\right) \mathbf{1}_{\{\rho=1 / 2\}} \stackrel{\mathcal{L}}{\longrightarrow} \mathcal{N}\left(0, \sigma_{X}^{2}\right),
$$


(iii) if $\frac{1}{2}<\rho<1$ then

$$
\frac{W_{n}-n \mu_{X}}{n^{\rho}} \stackrel{\mathrm{P}}{\rightarrow} \frac{K}{\mu_{Z}}\left(\mu_{Y}-\mu_{X}\right),
$$

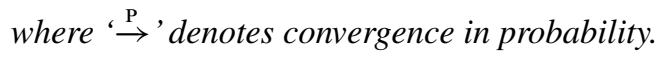

2. For $\mu_{X}=\mu_{Z}>0$, we have, almost surely,

$$
W_{n}=\mu_{X} n+o(n), \quad B_{n}=\frac{\mu_{X}^{2}}{\mu_{Y}} \frac{n}{\ln n}+o\left(\frac{n}{\ln n}\right) .
$$

Remark. Kotz et al. [14] gave exact formulae and some asymptotics for 2-type urns. They remarked that the

$$
\left(\begin{array}{ll}
1 & 0 \\
1 & 1
\end{array}\right)
$$

case gives asymptotics 'of an essentially different character'. They proved, 'heuristically', that $\mathrm{E}\left(B_{n}\right)$ is of order $n / \ln n$. This is expressed by part 2 of Theorem 2 .

An interesting exceptional case is when $Z \stackrel{\text { a.s. }}{=} 0$, where $\stackrel{\text { a.s., }}{=}$ denotes almost-sure equality. In this case the number of black balls remains unchanged, i.e. $B(t)=B_{0}$. The number, $W(t)$, of white balls in the branching process is a generalized Yule-type process with immigration. We obtain the following limit results.

Theorem 3. Consider a generalized Pólya urn with two colors and a triangular random replacement matrix

$$
\left(\begin{array}{ll}
X & 0 \\
Y & 0
\end{array}\right)
$$

Under hypotheses $(H)$, we have

1. $W_{n}=\mu_{X} n+o(n)$ almost surely,

2. if $\sigma_{X} \neq 0$ then we have the central limit theorem

$$
\frac{W_{n}-\mu_{X} n}{\sqrt{n}} \stackrel{\mathscr{L}}{\longrightarrow} \mathcal{N}\left(0, \sigma_{X}^{2}\right)
$$

3. if $X=\alpha \neq 0$ is nonrandom then

$$
\frac{1}{\sqrt{\ln n}}\left(W_{n}-n \alpha-\frac{\mu_{Y}-\alpha}{\alpha} B_{0} \ln n\right) \stackrel{\mathcal{L}}{\longrightarrow} \mathcal{N}\left(0,\left(\sigma_{Y}^{2}+\left(\mu_{Y}-\alpha\right)^{2}\right) \frac{B_{0}}{\alpha}\right) .
$$

Remarks. 1. The $\mu_{X}<\mu_{Z}$ case has been studied by Janson [11].

2. In (3), if $Y=\gamma=$ constant almost surely, we recover the result of Janson [12, Remark IV.4].

The next result deals with the diagonal case: $Y \stackrel{\text { a.s. }}{=} 0$.

Theorem 4. Consider a generalized Pólya urn with diagonal replacement matrix

$$
\left(\begin{array}{ll}
X & 0 \\
0 & Z
\end{array}\right)
$$

Let $\rho=\mu_{Z} / \mu_{X}$, and let $D=\mu_{X}^{\rho} B W^{-\rho}$. 
1. When $\mu_{X}>\mu_{Z}>0$,

(i) almost surely as $n \rightarrow+\infty$,

$$
W_{n}=\mu_{X} n+o(n), \quad B_{n}=D n^{\rho}+o\left(n^{\rho}\right),
$$

(ii) if $0<\rho \leq \frac{1}{2}$, we have, as $n \rightarrow+\infty$,

$$
\frac{W_{n}-n \mu_{X}}{\sqrt{n}}+\sqrt{\mu_{X}} \frac{\mu_{X}}{\mu_{Z}} B W^{-1 / 2} \mathbf{1}_{\{\rho=1 / 2\}} \stackrel{\mathcal{L}}{\longrightarrow} \mathcal{N}\left(0, \sigma_{X}^{2}\right),
$$

(iii) if $\frac{1}{2}<\rho<1$, we have, as $n \rightarrow+\infty$,

$$
\frac{W_{n}-n \mu_{X}}{n^{\rho}} \stackrel{\mathrm{P}}{\longrightarrow}-\mu_{X}^{\rho} \frac{\mu_{X}}{\mu_{Z}} B W^{-\rho} .
$$

2. When $\mu_{X}=\mu_{Z}$ almost surely as $n \rightarrow+\infty$,

$$
W_{n}=\mu_{X} \frac{W}{W+B} n+o(n), \quad B_{n}=\mu_{X} \frac{B}{W+B} n+o(n) .
$$

Remarks. 1. The branching process $(W(t), B(t))$ consists of two independent generalized Yule processes; thus, the limits $W$ and $B$ are independent and $\mathrm{P}(B>0) \mathrm{P}(W>0)>0$ (recall that $\left.\mu_{X} \mu_{Z}>0\right)$.

2. Let $f_{X}(s)=s \mathrm{E}\left(s^{X}\right)$ and $g_{Z}(s)=s \mathrm{E}\left(s^{Z}\right)$ be the probability generating functions of $X+1$ and $Z+1$, respectively, and let $\varphi_{W}(u)=\mathrm{E}\left[\mathrm{e}^{-u W}\right]$ and $\varphi_{B}(u)=\mathrm{E}\left[\mathrm{e}^{-u B}\right]$. Since the conditions $\mathrm{E}[X \ln X]<\infty$ and $\mathrm{E}[Z \ln Z]<\infty$ are fulfilled, we deduce from the results of [10] and [13] that

$$
\begin{array}{ll}
\varphi_{W}^{-1}(v)=(1-v) \exp \left(\int_{1}^{v}\left[\frac{\mu_{X}}{f_{X}(s)-s}-\frac{1}{s-1}\right] \mathrm{d} s\right), & 0<v \leq 1 \\
\varphi_{B}^{-1}(v)=(1-v) \exp \left(\int_{1}^{v}\left[\frac{\mu_{Z}}{g_{Z}(s)-s}-\frac{1}{s-1}\right] \mathrm{d} s\right), & 0<v \leq 1
\end{array}
$$

These two expressions give the characterization of $W$ 's and $B$ 's distributions. As a consequence, in case 2 of Theorem $4, W$ and $B$ have the same law.

In the deterministic case, where $X$ and $Z$ are constants, we find that $W$ and $B$ are beta distributed.

We also present some results about the splitting times $\left(\tau_{n}\right)_{n \geq 1}$. It is known (see [3, Section III.9]) that $\tau_{n}$ tends almost surely to $\infty$ as $n \rightarrow \infty$. We define, for all positive integers $n$, the process $\left\{\left(T_{n}, g_{n}\right)\right\}_{n \geq 1}$ by

$$
T_{n}=\tau_{n}-\sum_{j=0}^{n-1}\left(W\left(\tau_{j}\right)+B\left(\tau_{j}\right)\right)^{-1},
$$

where $g_{n}$ is the $\sigma$-algebra generated by $\tau_{1}, \ldots, \tau_{n}, W\left(\tau_{1}\right), B\left(\tau_{1}\right), \ldots, W\left(\tau_{n-1}\right)$, and $B\left(\tau_{n-1}\right)$. We have the following result.

Theorem 5. Under hypotheses $(H)$, the process $\left(T_{n}, g_{n}\right)_{n \geq 1}$ is an $L^{2}$-bounded martingale; consequently, it converges almost surely and in $L^{2}$ to some random variable $\left(T_{\infty}, g_{\infty}\right) \in L^{2}$. 
Using Theorem 5, we obtain an asymptotic expansion of $\tau_{n}$.

Proposition 1. Under hypotheses $(H)$, we have

$$
\frac{\tau_{n}}{\ln n}=\frac{1}{\mu_{X}}+o(1) \text { almost surely. }
$$

This extends the results of Athreya and Ney [3, Theorem V.7.3] and Janson [11, Lemma 11.1].

\section{Proof of Theorem 1}

Let

$$
f(t)=\mathrm{E}(W(t)), \quad g(t)=\mathrm{E}(B(t)), \quad \text { and } \quad \mathcal{H}(t)=\left(\begin{array}{c}
f(t) \\
g(t)
\end{array}\right)=\mathrm{E}(\mathcal{X}(t)) .
$$

The Markov property and the Chapman-Kolmogorov equation imply, this is a classical result, that

$$
\mathscr{H}^{\prime}(t)=\frac{\mathrm{d}}{\mathrm{d} t} \mathscr{H}(t)=A \mathscr{H}(t)
$$

and that $\mathcal{y}_{(t)}$ is an $\mathcal{F}_{t}$-martingale. By integration, (5) implies that

$$
\mathscr{H}(t)=\mathrm{e}^{t A} \mathscr{H}(0)=\mathrm{e}^{t A}\left(W_{0}, B_{0}\right)^{\top} .
$$

\section{1. $\mu_{X}>\mu_{Z}$ case}

Consider the processes $\{B(s), s \geq 0\}$ and $\{W(s), s \geq 0\}$ at time $t+\delta t$, where $\delta t$ is an infinitesimal increment of time. Given $B(t)$ and $W(t)$, the numbers of black and white balls at time $t+\delta t$ are equal to the numbers at time $t$ plus the numbers of black and white balls, respectively, added during the infinitesimal period $(t, t+\delta t)$. For each ball among the $S(t):=B(t)+W(t)$ black and white balls, the number of splittings that happen in the time interval $(t, t+\delta t)$ follows a $\mathcal{P}(\delta t)$-distribution; some of the resulting children may themselves produce children, but their number has an $O(\delta t)^{2}$ average and square mean. Furthermore, any two different balls have independent birth processes. Therefore, given $B(t)$ and $W(t), B(t+\delta t)$ and $W(t+\delta t)$ satisfy

$$
B(t+\delta t)=B(t)+\sum_{j=1}^{B(t)} \sum_{k=1}^{\mathcal{P}_{j}(\delta t)} Z_{k, j}+O(\delta t)^{2}
$$

and

$$
W(t+\delta t)=W(t)+\sum_{j=1}^{W(t)} \sum_{k=1}^{\mathcal{P}_{j}(\delta t)} X_{k, j}+\sum_{j=1}^{B(t)} \sum_{k=1}^{\mathcal{P}_{j}(\delta t)} Y_{k, j}+O(\delta t)^{2},
$$

where the $\mathcal{P}_{j}(\delta t)$ are independent and identically distributed (i.i.d.) random variables that have a $\mathcal{P}(\delta t)$-distribution, and $Z_{k, j}, X_{k, j}$, and $Y_{k, j}$ are i.i.d. random variables that respectively have $Z$ 's, $X$ 's, and $Y$ 's distribution and are independent of $B(t)$ and $W(t)$. Upon squaring (6) and (7) we obtain equations of the conditional second moments:

$$
\begin{aligned}
\mathrm{E}\left(B^{2}(t+\delta t) \mid B(t)\right)= & B^{2}(t)+2 B^{2}(t) \delta t \mu_{Z}+\mu_{Z^{2}} B(t) \delta t \\
& +\mu_{Z}^{2} \delta t B(t)(\delta t B(t)-1)+O\left((\delta t)^{2}\right)
\end{aligned}
$$


and

$$
\begin{aligned}
\mathrm{E}\left(W^{2}(t+\delta t) \mid B(t), W(t)\right)= & W^{2}(t)+2\left(W^{2}(t) \mu_{X}+W(t) B(t) \mu_{Y}\right) \delta t \\
& +\left(W(t) \mu_{X^{2}}+B(t) \mu_{Y^{2}}\right) \delta t+O\left((\delta t)^{2}\right) .
\end{aligned}
$$

Take expectations, and letting $\delta t \rightarrow 0$, we obtain

$$
\frac{\mathrm{d}}{\mathrm{d} t} \varphi(t)=2 \mu_{Z} \varphi(t)+g(t) \sigma_{Z}^{2}
$$

and

$$
\frac{\mathrm{d}}{\mathrm{d} t} \chi(t)=2 \mu_{X} \chi(t)+2 \mu_{Y} \psi(t)+\mu_{X^{2}} f(t)+\mu_{Y^{2}} g(t)
$$

where $\varphi(t)=\mathrm{E}\left(B^{2}(t)\right), \chi(t)=\mathrm{E}\left(W^{2}(t)\right)$, and $\psi(t)=\mathrm{E}(B(t) W(t))$. The solution of (8) is easily seen to be

$$
\varphi(t)=B_{0}\left(1+\frac{\sigma_{Z}^{2}}{\mu_{Z}}\right) \exp \left(2 \mu_{Z} t\right)-B_{0} \frac{\sigma_{Z}^{2}}{\mu_{Z}} \exp \left(\mu_{Z} t\right) .
$$

We prove similarly that $\psi(t)$ satisfies the differential equation

$$
\frac{\mathrm{d}}{\mathrm{d} t} \psi(t)=\left(\mu_{Z}+\mu_{X}\right) \psi(t)+\mu_{Y} \varphi(t) .
$$

After computation of $\psi(t)$ and $\chi(t)$, we easily conclude that $(\mathcal{y}(t), t \geq 0)$ is $L^{2}$-bounded.

\section{2. $\mu_{X}=\mu_{Z}$ case}

By (9), also available in this case, the process $\left(\exp \left(-t \mu_{X}\right) B(t), t \geq 0\right)$ is $L^{2}$-bounded. As in the first case, we prove that the process

$$
M(t):=\exp \left(-t \mu_{X}\right) W(t)-\mu_{Y} t \exp \left(-t \mu_{X}\right) B(t)
$$

is $L^{2}$-bounded.

\section{Proofs of the main results}

\subsection{Proof of Theorem 5}

For all integers $n \geq 1$, let $v_{n}=\tau_{n}-\tau_{n-1}$ be the holding time between the $(n-1)$ th and the $n$th splits and let $v_{0}=0$. Given $g_{n-1}, v_{n}$ is an exponential random variable with mean $\left(S_{n-1}\right)^{-1}$, where, for all $j \geq 0, S_{j}=W_{j}+B_{j}$. The martingale property follows from the fact that, for all $n \geq 1, \tau_{n}=\sum_{j=0}^{n} v_{j}$.

In the following we will prove that the process $\left(T_{n}, g_{n}\right)$ is $L^{2}$-bounded. Using Equation (4) of [3, p. 121], we have

$$
\mathrm{E}\left(T_{n}^{2}\right)=\sum_{j=0}^{n-1} \mathrm{E}\left(S_{j}^{-2}\right) .
$$

Define, for each $i \in \mathbb{N}, R_{i}=\min \left\{X_{i}, Y_{i}+Z_{i}\right\}$. The random variables $\left\{R_{i}\right\}_{\{i \geq 1\}}$ are independent with the same law as $R=\min \{X, Y+Z\}$.

By hypothesis (H1) we obtain

$$
\mathrm{P}(R>0)=\mathrm{P}(X>0) \mathrm{P}(Y+Z>0)>0 .
$$


Then

$$
\mu_{R}=\mathrm{E}(R)>0 \quad(\mathrm{P}(R=0)<1) .
$$

From Theorem III.9.4 of [3] and the strong law of large numbers, we obtain

$$
\lim _{n} \mathrm{E}\left(\frac{n^{2}}{\left(S_{0}+\sum_{i=1}^{n} R_{i}\right)^{2}}\right)=\frac{1}{\mu_{R}^{2}} .
$$

Since the initial total number of balls equals $S_{0}$, we obtain $S_{n} \geq S_{0}+\sum_{i=1}^{n} R_{i}$, so that

$$
\mathrm{E}\left(\frac{n^{2}}{S_{n}^{2}}\right) \leq \mathrm{E}\left(\frac{n^{2}}{\left(S_{0}+\sum_{i=1}^{n} R_{i}\right)^{2}}\right)
$$

Consequently, the sequence $\mathrm{E}\left(T_{n}^{2}\right)$ converges.

\subsection{Proof of Proposition 1}

We prove this proposition in two steps.

Step 1. Consider $\mu_{X}>\mu_{Z}$. For a continuous-time process $\&$ and a positive real $t_{0}$, we define

$$
\Delta \&\left(t_{0}\right)=\lim _{\varepsilon \rightarrow 0^{+}}\left[\delta\left(t_{0}+\varepsilon\right)-\delta\left(t_{0}-\varepsilon\right)\right] .
$$

We interpret $\Delta \&\left(t_{0}\right)$ as the jump at time $t_{0}$ of the process $(\delta(t))_{t \geq 0}$.

Let $S_{n}=W_{n}+B_{n}$. We can write, for $n \geq 1$,

$$
\begin{aligned}
\frac{S_{n}}{n} & =\frac{S_{0}}{n}+\frac{1}{n} \sum_{i=1}^{N_{n}^{\mathrm{W}}} \Delta W\left(\tau_{i}^{\mathrm{W}}\right)+\frac{1}{n} \sum_{i=1}^{N_{n}^{\mathrm{B}}}\left(\Delta B\left(\tau_{i}^{\mathrm{B}}\right)+\Delta W\left(\tau_{i}^{\mathrm{B}}\right)\right) \\
& =\frac{S_{0}}{n}+\frac{1}{n} \sum_{i=1}^{n} X_{i}+\frac{1}{n} \sum_{i=1}^{N_{n}^{\mathrm{B}}}\left(Z_{i}+Y_{i}-X_{i}\right) .
\end{aligned}
$$

From Theorem 1 and the almost-sure limit

$$
\lim _{n} \frac{B_{n}}{N_{n}^{\mathrm{B}}}=\mu_{Z}
$$

we can deduce the following lemma.

Lemma 1. If $\mu_{X}>\mu_{Z}>0$,

$$
N_{n}^{\mathrm{B}}=\frac{K}{\mu_{Z}} n^{\rho}+o\left(n^{\rho}\right) \text { almost surely, }
$$

where $\rho=\mu_{Z} / \mu_{X} \in(0,1)$ and $K$ is the random variable defined by

$$
K=\left(\mu_{X}\right)^{\rho} B\left(W+\frac{\mu_{Y}}{\mu_{X}-\mu_{Z}} B\right)^{-\rho} .
$$

Using this result and the strong law of large numbers, we deduce that

$$
\frac{1}{n} \sum_{i=1}^{n} X_{i}=\mu_{X}+o(1) \quad \text { and } \quad \frac{1}{n} \sum_{i=1}^{N_{n}^{\mathrm{B}}}\left(Z_{i}+Y_{i}-X_{i}\right)=O\left(n^{\rho-1}\right) \quad \text { almost surely }
$$


For large $n$, we can write

$$
\frac{n}{S_{n}}=\frac{1}{\mu_{X}}(1++o(1)) \quad \text { almost surely. }
$$

Using Theorem III.9.4 of [3], we conclude that

$$
\sum_{n \geq 1} \frac{1}{n}\left(\frac{n}{S_{n}}-\frac{1}{\mu_{X}}\right) \stackrel{\text { a.s. }}{=} C^{\prime}
$$

where $C^{\prime}$ is a finite random variable. This equality implies that, as $n \rightarrow+\infty$,

$$
\sum_{j=1}^{n-1}\left(\frac{1}{W_{j}+B_{j}}\right)-\frac{1}{\mu_{X}} \ln n \stackrel{\text { a.s. }}{=} O(1) .
$$

Using the fact that $T_{n}$ is an almost surely convergent martingale, and putting $T_{\infty}$ as the corresponding limit, it follows that

$$
\tau_{n}=\sum_{j=1}^{n-1} \frac{1}{S_{j}}+T_{\infty}+o(1)=\frac{\ln n}{\mu_{X}}+o(\ln n) \quad \text { almost surely. }
$$

Step 2. Consider $\mu_{X}=\mu_{Z}$. From (3) and (4), we prove that

$$
\lim _{t \rightarrow \infty} \frac{\exp \left(-\mu_{X} t\right) W(t)}{t} \stackrel{\text { a.s. }}{=} \mu_{Y} B .
$$

Replacing $t$ by $\tau_{n}$ in (4) and (10), we derive

$$
\lim _{n \rightarrow \infty} \frac{W_{n}}{\tau_{n} B_{n}} \stackrel{\text { a.s. }}{=} \mu_{Y} .
$$

We will prove at the end of Section 4.3 that

$$
\lim _{n \rightarrow \infty} \frac{W_{n}}{n}=\mu_{X} \quad \text { almost surely, }
$$

which implies that

$$
\lim _{n \rightarrow \infty} \frac{n}{\tau_{n} B_{n}} \stackrel{\text { a.s. }}{=} \frac{\mu_{Y}}{\mu_{X}} .
$$

Again, using (4), we conclude that

$$
\lim _{n \rightarrow \infty} \frac{n \exp \left(-\mu_{X} \tau_{n}\right)}{\tau_{n}} \stackrel{\text { a.s. }}{=} \frac{\mu_{Y}}{\mu_{X}} B .
$$

This means that

$$
\lim _{n \rightarrow \infty} \frac{\tau_{n}}{\ln n} \stackrel{\text { a.s. }}{=} \frac{1}{\mu_{X}} .
$$




\subsection{Proof of Theorem 2}

Part 1(i). By (1) and (2), we can write

$$
\frac{W(t)}{(B(t))^{\mu_{X} / \mu_{Z}}}+\frac{\mu_{Y}}{\mu_{X}-\mu_{Z}}\left(\exp \left(-t\left(\mu_{X}-\mu_{Z}\right)\right)-1\right)\left(\exp \left(-t \mu_{Z}\right) B(t)\right)^{1-\mu_{X} / \mu_{Z}} \stackrel{\text { a.s. }}{\rightarrow} \frac{W}{(B)^{\mu_{X} / \mu_{Z}}} \text {. }
$$

Replace $t$ by $\tau_{n}$ in (14); since $\left(W_{n}, B_{n}\right)=\left(W\left(\tau_{n}\right), B\left(\tau_{n}\right)\right)$ almost surely and since $\tau_{n}$ tends almost surely to $\infty$ as $n$ tends to $\infty$ (see [3, Section III.9]) we obtain

$$
\begin{aligned}
& \frac{W_{n}}{\left(B_{n}\right)^{\mu_{X} / \mu_{Z}}}+\frac{\mu_{Y}}{\mu_{X}-\mu_{Z}}\left(\exp \left(-\tau_{n}\left(\mu_{X}-\mu_{Z}\right)\right)-1\right)\left(\exp \left(-\tau_{n} \mu_{Z}\right) B_{n}\right)^{1-\mu_{X} / \mu_{Z}} \\
& \stackrel{\text { a.s. }}{\longrightarrow} \frac{W}{(B)^{\mu_{X} / \mu_{Z}}} .
\end{aligned}
$$

Using (11), we conclude that

$$
\frac{n \mu_{X}}{\left(B_{n}\right)^{\mu_{X} / \mu_{Z}}} \stackrel{\text { a.s. }}{\longrightarrow} \frac{\mu_{Y}}{\mu_{X}-\mu_{Z}} B^{\left(\mu_{Z}-\mu_{X}\right) / \mu_{Z}}+W B^{-\mu_{X} / \mu_{Z}} .
$$

This proves the second equation of 1(i). Then we deduce the fourth equation of 1(i) from the fact that $B_{n} / N_{n}^{\mathrm{B}} \stackrel{\text { a.s. }}{\longrightarrow} \mu_{Z}$. The third equation of 1(i) follows from the fact that $N_{n}^{\mathrm{W}}+N_{n}^{\mathrm{B}}=n$. Finally, we conclude the first equation of 1(i) using the fact that $W_{n} / N_{n}^{\mathrm{W}} \stackrel{\text { a.s. }}{\longrightarrow} \mu_{X}$

Part 1(ii). We have $W_{n}=W_{0}+\sum_{i=1}^{N_{n}^{\mathrm{W}}} X_{\tau_{i}^{\mathrm{W}}}+\sum_{j=1}^{N_{n}^{\mathrm{B}}} Y_{\tau_{i}^{\mathrm{B}}}$. Then

$$
\frac{W_{n}-n \mu_{X}}{\sqrt{n}}=\frac{1}{\sqrt{n}} \sum_{i=1}^{N_{n}^{\mathrm{W}}}\left(X_{\tau_{i}^{\mathrm{W}}}-\mu_{X}\right)+\frac{1}{\sqrt{n}} \sum_{j=1}^{N_{n}^{\mathrm{B}}}\left(Y_{\tau_{i}^{\mathrm{B}}}-\mu_{X}\right)+\frac{W_{0}}{\sqrt{n}} .
$$

With the help of the identity $N_{n}^{\mathrm{W}}=n+o(n)$ and the central limit theorem, we deduce that

$$
\frac{1}{\sqrt{n}} \sum_{i=1}^{N_{n}^{\mathrm{W}}}\left(X_{\tau_{i}^{\mathrm{W}}}-\mu_{X}\right) \stackrel{\mathcal{L}}{\longrightarrow} \mathcal{N}\left(0, \sigma_{X}^{2}\right) .
$$

Using 1(i), we deduce that, if $\rho<\frac{1}{2}$, almost surely,

$$
\lim _{n} \frac{1}{\sqrt{n}} \sum_{j=1}^{N_{n}^{\mathrm{B}}}\left(Y_{\tau_{i}^{\mathrm{B}}}-\mu_{X}\right)=\lim _{n} \frac{N_{n}^{\mathrm{B}}}{\sqrt{n}} \frac{1}{N_{n}^{\mathrm{B}}} \sum_{j=1}^{N_{n}^{\mathrm{B}}}\left(Y_{\tau_{i}^{\mathrm{B}}}-\mu_{X}\right)=0 \times\left(\mu_{Y}-\mu_{X}\right)=0
$$

and, if $\rho=\frac{1}{2}$,

$$
\begin{aligned}
& \frac{1}{\sqrt{n}} \sum_{j=1}^{N_{n}^{\mathrm{B}}}\left(Y_{\tau_{i}^{\mathrm{B}}}-\mu_{X}\right)-\frac{K}{m_{Z}}\left(\mu_{Y}-\mu_{X}\right) \\
& \quad=\frac{1}{\sqrt{n}} \sum_{j=1}^{N_{n}^{\mathrm{B}}}\left(Y_{\tau_{i}^{\mathrm{B}}}-\mu_{Y}\right)+N_{n}^{\mathrm{B}}\left(\mu_{Y}-\mu_{X}\right) \\
& \quad=\frac{N_{n}^{\mathrm{B}}}{\sqrt{n}} \frac{1}{N_{n}^{\mathrm{B}}} \sum_{j=1}^{N_{n}^{\mathrm{B}}}\left(Y_{\tau_{i}^{\mathrm{B}}}-\mu_{Y}\right)+\left(\frac{N_{n}^{\mathrm{B}}}{\sqrt{n}}-\frac{K}{m_{Z}}\right)\left(\mu_{Y}-\mu_{X}\right) .
\end{aligned}
$$


The desired result can be established using the facts that

$$
\left(\frac{N_{n}^{\mathrm{B}}}{\sqrt{n}}-\frac{K}{m_{Z}}\right) \stackrel{\text { a.s. }}{\rightarrow} 0 \text { and } \quad \frac{1}{N_{n}^{\mathrm{B}}} \sum_{j=1}^{N_{n}^{\mathrm{B}}}\left(Y_{\tau_{i}^{\mathrm{B}}}-\mu_{Y}\right) \stackrel{\text { a.s. }}{\rightarrow} 0 .
$$

Part 1(iii). In this case we have $\frac{1}{2}<\rho<1$. Splitting $W_{n}$ as in 1(ii),

$$
\frac{W_{n}-n \mu_{X}}{n^{\rho}}=\frac{1}{n^{\rho}} \sum_{i=1}^{N_{n}^{\mathrm{W}}}\left(X_{\tau_{i}^{\mathrm{W}}}-\mu_{X}\right)+\frac{1}{n^{\rho}} \sum_{j=1}^{N_{n}^{\mathrm{B}}}\left(Y_{\tau_{i}^{\mathrm{B}}}-\mu_{Y}\right)+\frac{N_{n}^{\mathrm{B}}}{n^{\rho}}\left(\mu_{Y}-\mu_{X}\right)+\frac{W_{0}}{n^{\rho}} .
$$

The first term on the right-hand side tends in $L^{2}$ to 0 :

$$
\mathrm{E}\left(\frac{1}{n^{\rho}} \sum_{i=1}^{N_{n}^{\mathrm{W}}}\left(X_{\tau_{i}^{\mathrm{W}}}-\mu_{X}\right)\right)^{2}=\sigma_{X}^{2} \mathrm{E}\left(\frac{N_{n}^{\mathrm{W}}}{n^{2 \rho}}\right) \leq \sigma_{X}^{2} n^{1-2 \rho} \rightarrow 0 .
$$

By the strong law of large numbers and 1(i), we conclude that

$$
\frac{1}{n^{\rho}} \sum_{j=1}^{N_{n}^{\mathrm{B}}}\left(Y_{\tau_{i}^{\mathrm{B}}}-\mu_{Y}\right)=\frac{N_{n}^{\mathrm{B}}}{n^{\rho}} \frac{1}{N_{n}^{\mathrm{B}}} \sum_{j=1}^{N_{n}^{\mathrm{B}}}\left(Y_{\tau_{i}^{\mathrm{B}}}-\mu_{Y}\right) \stackrel{\text { a.s. }}{\longrightarrow} 0 \quad \text { and } \quad \frac{N_{n}^{\mathrm{B}}}{n^{\rho}}\left(\mu_{Y}-\mu_{X}\right) \stackrel{\text { a.s. }}{\longrightarrow} \frac{K}{\mu_{Z}} \text {. }
$$

Part 2. It follows from (3) and (4) that

$$
\mu_{X} \frac{W_{n}}{B_{n}}-\mu_{Y} \ln \left(B_{n}\right) \stackrel{\text { a.s. }}{\longrightarrow} \mu_{X} \frac{W}{B}-\mu_{Y} \ln (B) .
$$

This leads to the following result.

Lemma 2. Under the conditions of part 2 of Theorem 2, we have, almost surely,

$$
\lim _{n \rightarrow \infty} \frac{W_{n}}{B_{n}}=\infty, \quad N_{n}^{\mathrm{W}}=n+o(n), \quad \text { and } \quad N_{n}^{\mathrm{B}}=o(n) .
$$

Proof. Because of (2), $B(t) \sim B \exp \left(t \mu_{Z}\right)$ when $t$ tends to $\infty$, so that $B_{n}$ tends to $\infty$ when $n$ tends to $\infty$. Thus, (16) implies that $W_{n} / B_{n}$ tends almost surely to $\infty$ as $n$ tends to $\infty$. But, for $n \geq 1$,

$$
W_{n}=W_{0}+\sum_{i=1}^{N_{n}^{\mathrm{W}}} X_{\tau_{i}^{\mathrm{W}}}+\sum_{j=1}^{N_{n}^{\mathrm{B}}} Y_{\tau_{j}^{\mathrm{B}}} \quad \text { and } \quad B_{n}=B_{0}+\sum_{j=1}^{N_{n}^{\mathrm{B}}} Z_{\tau_{j}^{\mathrm{B}}},
$$

so $B_{n} / N_{n}^{\mathrm{B}} \stackrel{\text { a.s. }}{\longrightarrow} \mu_{Z}$ and the first assertion of this lemma yields $W_{n} / N_{n}^{\mathrm{B}} \stackrel{\text { a.s. }}{\longrightarrow} \infty$. This implies that

$$
\frac{N_{n}^{\mathrm{W}}}{N_{n}^{\mathrm{B}}} \stackrel{\text { a.s. }}{\rightarrow} \infty \text {. }
$$

The result of Lemma 2 follows using the identity $N_{n}^{\mathrm{W}}+N_{n}^{\mathrm{B}}=n$.

By the strong law of large numbers and Lemma 2, we conclude that

$$
\lim _{n \rightarrow \infty} \frac{W_{n}}{n}=\mu_{X} \quad \text { almost surely. }
$$

The asymptotic of $B_{n}$ can be easily obtained using (12) and (13). 


\subsection{Proof of Theorem 3}

To prove Theorem 3, we first establish a corollary, which follows from Theorem 3, and a lemma, which follows from (19) and (21), below.

Corollary 1. The process $\left(\exp \left(-\mu_{X} t\right)\left(W(t)+\mu_{Y} / \mu_{X}\right)\right)_{(t \geq 0)}$ is a positive martingale. Let

$$
\lim _{t \rightarrow \infty} \exp \left(-\mu_{X} t\right)\left(W(t)+\frac{\mu_{Y}}{\mu_{X}}\right)=W+\frac{\mu_{Y}}{\mu_{X}} .
$$

In particular,

$$
\mathrm{E}(W(t))=\frac{\mu_{Y}+\mu_{X}}{\mu_{X}} \exp \left(t \mu_{X}\right)-\frac{\mu_{Y}}{\mu_{X}} .
$$

Lemma 3. Almost surely, as $n \rightarrow+\infty$,

$$
\frac{N\left(\tau_{n}\right)}{\ln n}=\frac{B_{0}}{\mu_{X}}+o(1) \text { and } \tau_{n}=\frac{\ln n}{\mu_{X}}+O(1) .
$$

Proof of Theorem 3. Part 1. For $t \geq 0$, denote by $N(t):=\#\left\{k: \tau_{k}^{\mathrm{B}} \leq t\right\}$ the number of splits of black balls up to time $t$, and denote by $\tau_{n}$ the time of the $n$th draw. Then

$$
W\left(\tau_{n}\right)=W_{n}=W_{0}+\sum_{j=1}^{n-N\left(\tau_{n}\right)} X_{\tau_{j}^{\mathrm{W}}}+\sum_{i=1}^{N\left(\tau_{n}\right)} Y_{\tau_{i}^{\mathrm{B}}}
$$

Since $(N(t))_{(t \geq 0)}$ is a Poisson process with intensity $B_{0}$,

$$
\frac{N(t)}{t} \stackrel{\text { a.s. }}{\longrightarrow} B_{0} \quad \text { as } t \rightarrow \infty .
$$

In particular, $\exp \left(-\mu_{X} t\right) N(t) \stackrel{\text { a.s. }}{\longrightarrow} 0$ as $t \rightarrow \infty$.

Then, from (17) and (18) and the fact that $\tau_{n} \stackrel{\text { a.s. }}{\longrightarrow} \infty$, we deduce that

$$
\begin{aligned}
& \lim _{n} \exp \left(-\mu_{X} \tau_{n}\right) W_{n}=W+\frac{\mu_{Y}}{\mu_{X}}, \\
& \lim _{n} \mu_{X} \exp \left(-\mu_{X} \tau_{n}\right) n=W+\frac{\mu_{Y}}{\mu_{X}} .
\end{aligned}
$$

Equation (20) can be written as

$$
\exp \left(-\mu_{X} \tau_{n}\right) W_{n}=n \mu_{X} \exp \left(-\mu_{X} \tau_{n}\right) \frac{W_{n}}{n \mu_{X}} \stackrel{\text { a.s. }}{\rightarrow} W+\frac{\mu_{Y}}{\mu_{X}} .
$$

Consequently, by (21), we conclude that

$$
\frac{W_{n}}{n \mu_{X}} \stackrel{\text { a.s. }}{\longrightarrow} 1 \text {. }
$$

Part 2. We have the following representation of $W_{n}$ :

$$
W_{n}=W_{0}+\sum_{k=1}^{n} X_{k}+\sum_{j=1}^{N\left(\tau_{n}\right)}\left(Y_{\tau_{j}^{\mathrm{B}}}-X_{\tau_{j}^{\mathrm{B}}}\right)
$$


where the $\left(X_{i}\right)_{1 \leq i \leq n}$ and the $\left(Y_{j}\right)_{j \geq 1}$ are mutually i.i.d. as $X$ and $Y$, respectively, and $\tau_{1}^{\mathrm{B}}, \ldots$, $\tau_{N\left(\tau_{n}\right)}^{\mathrm{B}}$ are the orders of the black ball splits up to time $\tau_{n}$.

Using Lemma 3 and the strong law of large numbers, the process

$$
\Delta(t):=\frac{1}{N(t)} \sum_{j=1}^{N(t)}\left(Y_{\tau_{j}^{\mathrm{B}}}-X_{\tau_{j}^{\mathrm{B}}}\right)
$$

converges almost surely to $\mu_{Y}-\mu_{X}$. The remainder of the proof of part 2 follows from the identity

$$
W_{n}-n \mu_{X}-W_{0}=\sum_{k=1}^{n}\left(X_{k}-\mu_{X}\right)+N\left(\tau_{n}\right) \Delta\left(\tau_{n}\right),
$$

the central limit theorem, Lemma 3, and Slutsky's theorem.

Part 3. Since $(N(t))_{(t \geq 0)}$ is a Poisson process with intensity $B_{0}$ and $\tau_{n} \rightarrow \infty$ when $n \rightarrow \infty$, we have

$$
\frac{N\left(\tau_{n}\right)-B_{0} \tau_{n}}{\sqrt{\tau_{n}}} \stackrel{\mathcal{L}}{\longrightarrow} \mathcal{N}\left(0, B_{0}\right) .
$$

Consider

$$
\begin{aligned}
K_{n} & =\frac{1}{\sqrt{\ln n}}\left(W_{n}-n \alpha-\frac{\mu_{Y}-\alpha}{\alpha} B_{0} \ln n\right) \\
& =\frac{1}{\sqrt{\ln n}}\left(N\left(\tau_{n}\right)\left(\mu_{Y}-\alpha\right)-\frac{\mu_{Y}-\alpha}{\alpha} B_{0} \ln n+\sum_{j=1}^{N\left(\tau_{n}\right)}\left(Y_{\tau_{j}^{\mathrm{B}}}-\mu_{Y}\right)\right) \\
& =\left(\mu_{Y}-\alpha\right) \frac{N\left(\tau_{n}\right)-\left(B_{0} / \alpha\right) \ln n}{\sqrt{\ln n}}+\frac{1}{\sqrt{\ln n}} \sum_{j=1}^{N\left(\tau_{n}\right)}\left(Y_{\tau_{j}^{\mathrm{B}}}-\mu_{Y}\right) .
\end{aligned}
$$

Let, for $t \in(-1,1), \phi_{n}(t)=\mathrm{E}\left(\exp \left(-t K_{n}\right)\right)$ denote the Laplace transform of $K_{n}$ and $\phi(t)=$ $\mathrm{E}\left(\exp \left(-t\left(Y-\mu_{Y}\right)\right)\right)$ denote the Laplace transform of $Y-\mu_{Y}$. We have

$$
\begin{aligned}
\phi_{n}(t)= & \mathrm{E}\left(\exp \left(-t\left(\mu_{Y}-\alpha\right) \frac{N\left(\tau_{n}\right)-\left(B_{0} / \alpha\right) \ln n}{\sqrt{\ln n}}\right) \exp \left(-\frac{t}{\sqrt{\ln n}} \sum_{j=1}^{N\left(\tau_{n}\right)}\left(Y_{\tau_{j}^{\mathrm{B}}}-\mu_{Y}\right)\right)\right) \\
= & \mathrm{E}\left(\exp \left(-t\left(\mu_{Y}-\alpha\right) \frac{N\left(\tau_{n}\right)-\left(B_{0} / \alpha\right) \ln n}{\sqrt{\ln n}}\right)\left(\phi\left(\frac{t}{\sqrt{\ln n}}\right)\right)^{N\left(\tau_{n}\right)}\right) \\
= & \mathrm{E}\left(\exp \left(-t\left(\mu_{Y}-\alpha\right) \frac{N\left(\tau_{n}\right)-\left(B_{0} / \alpha\right) \ln n}{\sqrt{\ln n}}\right) \exp \left(N\left(\tau_{n}\right) \ln \left(\phi\left(\frac{t}{\sqrt{\ln n}}\right)\right)\right)\right) \\
= & \mathrm{E}\left(\exp \left(\frac{N\left(\tau_{n}\right)-\left(B_{0} / \alpha\right) \ln n}{\sqrt{\ln n}}\left(\sqrt{\ln n} \ln \left(\phi\left(\frac{t}{\sqrt{\ln n}}\right)\right)-t\left(\mu_{Y}-\alpha\right)\right)\right)\right) \\
& \times \exp \left(\frac{B_{0}}{\alpha} \ln n \ln \left(\phi\left(\frac{t}{\sqrt{\ln n}}\right)\right)\right) .
\end{aligned}
$$

For large $n$, we have

$$
\begin{aligned}
\phi\left(\frac{t}{\sqrt{\ln n}}\right) & =\mathrm{E}\left(1-\frac{t}{\sqrt{\ln n}}\left(Y-\mu_{Y}\right)+\frac{t^{2}}{2 \ln n}\left(Y-\mu_{Y}\right)^{2}+o\left(\frac{1}{\ln n}\right)\right) \\
& =1+\frac{t^{2}}{2 \ln n} \sigma_{Y}^{2}+o\left(\frac{1}{\ln n}\right) .
\end{aligned}
$$


It then follows that

$$
\frac{B_{0}}{\alpha} \ln n \ln \left(\phi\left(\frac{t}{\sqrt{\ln n}}\right)\right)=\frac{B_{0}}{2 \alpha} t^{2} \sigma_{Y}^{2}+o(1)
$$

Consequently,

$$
\exp \left(\frac{B_{0}}{\alpha} \ln n \ln \left(\phi\left(\frac{t}{\sqrt{\ln n}}\right)\right) \longrightarrow \exp \left(\frac{B_{0}}{2 \alpha} \sigma_{Y}^{2} t^{2}\right)\right) \quad \text { as } n \rightarrow \infty .
$$

From Lemma 3 and (22), we derive

$$
\begin{aligned}
\frac{N\left(\tau_{n}\right)-\left(B_{0} / \alpha\right) \ln n}{\sqrt{\ln n}} & =\frac{N\left(\tau_{n}\right)-B_{0} \tau_{n}}{\sqrt{\ln n}}+\frac{B_{0}\left(\tau_{n}-\ln n / \alpha\right)}{\sqrt{\ln n}} \\
& =\frac{N\left(\tau_{n}\right)-B_{0} \tau_{n}}{\sqrt{\tau_{n}}} \sqrt{\frac{\tau_{n}}{\ln n}}+\frac{B_{0}\left(\tau_{n}-\ln n / \alpha\right)}{\sqrt{\ln n}} \\
& \stackrel{\mathcal{L}}{\longrightarrow} \mathcal{N}\left(0, \frac{B_{0}}{\alpha}\right) .
\end{aligned}
$$

Then, when $n \rightarrow \infty$, it follows that

$$
\sqrt{\ln n} \ln \left(\phi\left(\frac{t}{\sqrt{\ln n}}\right)\right)=\sqrt{\ln n}\left(\frac{t^{2}}{2 \ln n} \sigma_{Y}^{2}+o\left(\frac{1}{\ln n}\right)\right) \rightarrow 0 .
$$

Finally, we conclude that

$$
\phi_{n}(t) \rightarrow \exp \left(\frac{B_{0}}{2 \alpha}\left(\mu_{Y}-\alpha\right)^{2} t^{2}+\frac{B_{0}}{2 \alpha} \sigma_{Y}^{2} t^{2}\right) .
$$

\subsection{Proof of Theorem 4}

We study the asymptotic behavior of the numbers of black balls, $B(t)$, and white balls, $W(t)$. We see that $(B(t))_{(t \geq 0)}$ and $(W(t))_{(t \geq 0)}$ are independent Yule-type branching processes. Let $\mathcal{F}_{t}^{W}$ and $\mathcal{F}_{t}{ }^{B}$ be the sigma fields generated by the processes $(W(u), 0 \leq u \leq t)$ and $(B(u), 0 \leq u \leq t)$, respectively. We deduce from Theorem 1 that

$$
\mathrm{E}(B(t))=B_{0} \exp \left(\mu_{Z} t\right), \quad \mathrm{E}(W(t))=W_{0} \exp \left(\mu_{X} t\right),
$$

and

$$
\begin{gathered}
\mathrm{E}\left(W(t+s) \mid \mathcal{F}_{t}^{W}\right)=\exp \left(\mu_{X} s\right) W(t), \\
\mathrm{E}\left(B(t+s) \mid \mathcal{F}_{t}^{B}\right)=\exp \left(\mu_{Z} s\right) B(t), \\
\exp \left(-\mu_{X} t\right) W(t) \stackrel{\text { a.s. }}{\longrightarrow} W, \quad \exp \left(-\mu_{Z} t\right) B(t) \stackrel{\text { a.s. }}{\longrightarrow} B .
\end{gathered}
$$

By (23) we conclude that

$$
\frac{W_{n}^{\rho}}{B_{n}} \stackrel{\text { a.s. }}{\longrightarrow} \frac{W^{\rho}}{B} .
$$

Part 1(i) of Theorem 4 can be derived from (24) and the following lemma.

Lemma 4. If $\mu_{X} \times \mu_{Z}>0$ then

$$
\frac{W_{n}}{\mu_{X}}+\frac{B_{n}}{\mu_{Z}} \stackrel{\text { a.s. }}{=} n+o(n) \quad(n \rightarrow \infty)
$$


Proof. We have $W_{n}=W_{0}+\sum_{k=1}^{N_{n}^{\mathrm{W}}} X_{\tau_{k}^{\mathrm{W}}}$ and $B_{n}=B_{0}+\sum_{k=1}^{N_{n}^{\mathrm{B}}} Z_{\tau_{k}^{\mathrm{B}}}$.

By the strong law of large numbers, and the fact that

$$
\lim _{n \rightarrow \infty} N_{n}^{\mathrm{W}}=\infty, \quad \lim _{n \rightarrow \infty} N_{n}^{\mathrm{B}}=\infty, \quad \text { almost surely, }
$$

we deduce that

$$
\frac{W_{n}}{N_{n}^{\mathrm{W}}} \stackrel{\text { a.s. }}{\longrightarrow} \mu_{X}, \quad \frac{B_{n}}{N_{n}^{\mathrm{B}}} \stackrel{\text { a.s. }}{\longrightarrow} \mu_{Z} .
$$

This implies, almost surely, that

$$
\frac{W_{n}}{\mu_{X}} \simeq N_{n}^{\mathrm{W}}+o(n), \quad \frac{B_{n}}{\mu_{Z}} \simeq N_{n}^{\mathrm{B}}+o(n) .
$$

Using the fact that $N_{n}^{\mathrm{B}}+N_{n}^{\mathrm{W}}=n$, and (25), we obtain the desired expansion. To prove part 1(ii), observe from (25) and part 1(i) that

$$
N_{n}=n+o(n), \quad N_{n}^{\mathrm{B}}=\frac{D}{\mu_{Z}} n^{\rho}+o\left(n^{\rho}\right) .
$$

For $n \geq 1, W_{n}$ can be decomposed as

$$
\frac{W_{n}-n \mu_{X}}{\sqrt{n}}=\frac{W_{0}}{\sqrt{n}}+\frac{1}{\sqrt{n}} \sum_{k=1}^{n}\left(X_{k}-\mu_{X}\right)-\frac{1}{\sqrt{n}} \sum_{j=1}^{N_{n}^{\mathrm{B}}} X_{\tau_{j}^{\mathrm{B}}} .
$$

By the central limit theorem we have

$$
\frac{1}{\sqrt{n}} \sum_{k=1}^{n}\left(X_{k}-\mu_{X}\right) \stackrel{\mathscr{L}}{\longrightarrow} N\left(0, \sigma_{X}^{2}\right) .
$$

Using (26) and the strong law of large numbers, if $\rho<\frac{1}{2}$,

$$
\frac{1}{\sqrt{n}} \sum_{j=1}^{N_{n}^{\mathrm{B}}} X_{\tau_{j}^{\mathrm{B}}}=\frac{N_{n}^{\mathrm{B}}}{\sqrt{n}} \frac{1}{N_{n}^{\mathrm{B}}} \sum_{j=1}^{N_{n}^{\mathrm{B}}} X_{\tau_{j}^{\mathrm{B}}} \stackrel{\text { a.s. }}{\longrightarrow} 0 \times \mu_{X}=0
$$

and, if $\rho=\frac{1}{2}$,

$$
\frac{D}{\mu_{X}} \mu_{Z}-\frac{1}{\sqrt{n}} \sum_{j=1}^{N_{n}^{\mathrm{B}}} X_{\tau_{j}^{\mathrm{B}}}=\frac{D}{\mu_{X}} \mu_{Z}-\frac{N_{n}^{\mathrm{B}}}{\sqrt{n}} \frac{1}{N_{n}^{\mathrm{B}}} \sum_{j=1}^{N_{n}^{\mathrm{B}}} X_{\tau_{j}^{\mathrm{B}}} \stackrel{\text { a.s. }}{\longrightarrow} 0 .
$$

Part 1(ii) now follows using Slutsky's theorem.

To prove part 1(iii), observe that

$$
\frac{W_{n}-n \mu_{X}}{n^{\rho}}=\frac{W_{0}}{n^{\rho}}+\frac{1}{n^{\rho}} \sum_{k=1}^{n}\left(X_{k}-\mu_{X}\right)-\frac{1}{n^{\rho}} \sum_{j=1}^{N_{n}^{\mathrm{B}}} X_{\tau_{j}^{\mathrm{B}}} .
$$

From (15),

$$
\frac{1}{n^{\rho}} \sum_{k=1}^{n}\left(X_{k}-\mu_{X}\right) \stackrel{L^{2}}{\longrightarrow} 0
$$


where ' $\stackrel{L^{2}}{\longrightarrow}$ ' denotes $L^{2}$-convergence, and, by (26),

$$
\frac{1}{n^{\rho}} \sum_{j=1}^{N_{n}^{\mathrm{B}}} X_{\tau_{j}^{\mathrm{B}}} \stackrel{\text { a.s. }}{\longrightarrow}-\frac{D \mu_{X}}{\mu_{Z}} .
$$

We now prove part 2. Since $\tau_{n} \rightarrow+\infty$, we deduce from (23) that

$$
\frac{W_{n}}{B_{n}} \stackrel{\text { a.s. }}{\longrightarrow} \frac{W}{B} \text { if } n \rightarrow \infty \text {. }
$$

Using the relation $N_{n}^{\mathrm{W}}+N_{n}^{\mathrm{B}}=n$, and the facts that $N_{n}^{\mathrm{B}} \rightarrow \infty$ and $N_{n}^{\mathrm{W}} \rightarrow \infty$, we deduce from (27) that, almost surely,

$$
N_{n}^{\mathrm{B}}=\frac{B}{B+W} n+o(n) \quad \text { and } \quad N_{n}^{\mathrm{W}}=\frac{W}{B+W} n+o(n),
$$

which concludes the proof of part 2 .

\section{Acknowledgements}

I thank Svante Janson, Hosam Mahmoud, and Nabil Lasmar for helpful discussions. Thanks are also due to Nicolas Pouyanne and Brigitte Chauvin for judicious remarks. I thank an anonymous referee for a very careful reading of the paper, and for comments, suggestions, and remarks that improved the presentation of the results.

\section{References}

[1] Aldous, D., Flannery, B. And Palacios, J. L. (1988). Two applications of urn processes: the fringe analysis of search trees and the simulation of quasi-stationary distributions of Markov chains. Prob. Eng. Inf. Sci. 2, 293-307.

[2] Athreya, K. B. and Karlin, S. (1968). Embedding of urn schemes into continuous time Markov branching process and related limit theorems. Ann. Math. Statist. 39, 1801-1817.

[3] Athreya, K. B. And Ney, P. E. (1972). Branching Processes. Springer, New York.

[4] Bagchi, A. And Pal, A. K. (1985). Asymptotic normality in the generalized Pólya-Eggenberger urn model, with an application to computer data structures. SIAM J. Algebraic Discrete Meth. 6, 394-405.

[5] Bernstein, S. (1940). Sur un probléme du schéma des urnes à composition variable. C. R. (Doklady) Acad. Sci. URSS 28, 5-7.

[6] Eggenberger, F. and Pòlya, G. (1923). Über die statistik verketetter vorgäge. Z. Angew. Math. Mech. 1, 279-289.

[7] Flajolet, P. and Vincent, P. (2005). Triangular urns with dimension 2 and 3. Doctoral Thesis, École Polytechnique.

[8] Flajolet, P., Gabarró, J. and Pekari, H. (2005). Analytic urns. Ann. Prob. 33, 1200-1233.

[9] Gouet, R. (1993). Martingale functional central limit theorems for a generalized Pólya urn. Ann. Prob. 21, $1624-1639$.

[10] Harris, T. E. (1951). Some mathematical models for branching processes. In Proc. 2nd Berkeley Symp. Math. Statist. Prob., University of California Press, Berkeley, pp. 305-328.

[11] Janson, S. (2004). Functional limit theorems for multitype branching processes and generalized Pólya urns. Stoch. Process. Appl. 110, 177-245.

[12] Janson, S. (2006). Limit theorems for triangular urn schemes. Prob. Theory Relat. Fields 134, 417-452.

[13] Karlin, S. and McGregor, J. (1968). Embeddability of discrete time simple branching processes into continuous time branching processes. Trans. Amer. Math. Soc. 132, 115-136.

[14] Kotz, S., Mahmoud, H. and Robert, P. (2000). On generalized Pólya urn models. Statist. Prob. Lett. 49, 163-173.

[15] Pólya, G. (1931). Sur quelques points de la théorie des probabilités. Ann. Inst. H. Poincaré 1, 117-161.

[16] Savkevitch, V. (1940). Sur le schéma des urnes à composition variable. C. R (Doklady) Acad. Sci. URSS 28, $8-12$. 